\title{
The Timing of Lexical Memory Retrievals in Language Production Working paper in progress
}

\author{
Jeremy Cole, David Reitter \\ College of Information Sciences and Technology \\ The Pennsylvania State University \\ University Park, PA
}

\begin{abstract}
This paper explores the time course of lexical memory retrieval by modeling fluent language production. The duration of retrievals is predicted using the ACT-R cognitive architecture. In a large-scale observational study of a spoken corpus, we find that language production at a time point preceding a word is sped up or slowed down depending on activation of that word. This computational analysis has consequences for the theoretical model of language production. The data point to interference between lexical and phonological stages as well as a quantifiable buffer for lexical information.
\end{abstract}

\section{Introduction}

Speech varies greatly in fluency, and some of its speed variation can be traced to the utterance spoken (Jespersen, 1992). Low-frequency words (e.g., Bell et al., 2009) are well known to slow down speech, for example. Variables correlated with fluency give valuable cues to the architecture of the language processing system. A computational-cognitive model, however, has yet to emerge.

In this paper, we propose a cognitive model, in which lexical memory retrievals may explain some of the variability in speech rates. In particular, frequency, context and recent uses together have the potential to quantify retrieval delays through activation (Anderson, 1991). Activation, in its most common usage, refers to the way nodes in semantic networks become easier to retrieve after adjacent nodes have been activated, typically through a presentation (Collins and Loftus, 1975). In particular, activation makes a direct claim that more highly activated words require less time to retrieve, and vice versa (Anderson, 1983).

The language production process as a whole likely requires some amount of both serial and sequential processing. For instance, the standard model proposes that an idea is generated, grammatically encoded, and only then phonologically 
encoded (Bock and Levelt, 2002). Still, most models of language production presuppose some amount of planning of output (e.g. Pickering and Garrod, 2013), so we could instead divide language production into planning this output and the actual process of outputting. The overlap and relationship of these processes is not fully understood, but given that most output is likely planned, the scale at which the planning takes place and the amount of time between planned output and the actual process of outputting remains unclear. However, if interactions between processes are observed, then we can likewise see when they overlap in time.

To summarize, we are suggesting that some of the variance in speech rate is not due to the linguistic properties of the words currently or about to be outputted, but the words still in the planning phase. We propose a model that uses a buffer of several words between initial retrieval and output, during which grammatical and morphological encoding take place. We examine this by calculating retrieval activation for a word and evaluating the influence of that activation on the empirical speech timing in the Switchboard corpus several words beforehand. The effect of activation is distributed over preceding words in a way that is characteristic of a shared-resource, buffer-based account of language production.

\section{Related Work}

\subsection{Stages of Language Production}

Grammatical encoding can be divided into functional and positional processing steps (Bock and Levelt, 2002). The functional step selects lexical items and assigns functions, while the positional step then combines the items to produces constituents. In our account, we expect that these mutually dependent steps work in parallel.

An important early part of functional processing retrieves lexical information, which we will examine in this paper. We evaluate the consequences of lexical access, which is assumed to be affected by the cost associated with any retrieval from declarative memory. Much discussion in this area has concerned the question whether lexical access happens in a single stage (Dell et al., 1997) or in multiple stages and overlaps with grammatical encoding (Caramazza, 1997; Roelofs et al., 1998; Caramazza, 2006). Here, we follow ACT-R's serial and partially symbolic nature, which in turn leads to some theoretical commitments to non-parallel processing: language production is staged and discrete. Nonetheless, each stage can be composed of several steps, and steps from syntactic and phonological processing likely interleave. This is compatible with empirical findings and the overall theoretical debate (Ferreira and Slevc, 2007). The precise timeline of processing is unclear, but as we will argue in this paper, large-scale speech data can give us usable clues to that effect.

\subsection{Incrementality in Language Production}

The second issue we address concerns the timing of memory retrievals, which is also related to the idea of incremental processing. It is a commonly implied 


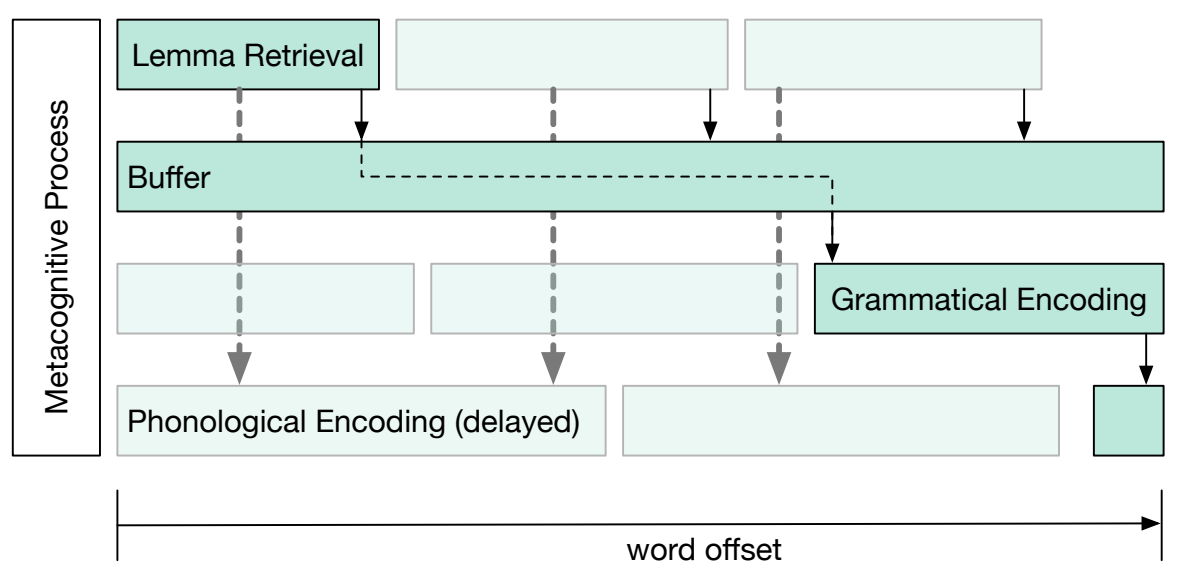

Figure 1: Our psychological model assumes that language production involves several parallel processes, and that retrieval of lemmas interferes with concurrent retrieval and/or encoding of phonological representations (dashed arrows) due to concurrent resource usage. Lemmas are retrieved several words before they are spoken. Their exact point of retrieval could depend on other factors.

assumption that language processing proceeds incrementally. In grammatical encoding, this property concerns when and in which order syntactic choices are made. For instance, all of them could be made before phonological processing starts (non-incremental case), or they could be made in order as necessary. Existing high-level models of language production proceed incrementally at various steps in a chain of content selection, aggregation and sentence realization (e.g. Bock and Levelt, 2002; Guhe, 2007).

Ferreira (1996) makes an argument for incrementality, based on the observation that competitive syntactic alternatives facilitate production rather than making it more difficult. An incremental account of sentence realization would predict such an effect, as syntactic "flexibility" introduced by the alternatives makes it easier to find a workable syntactic decision. By contrast, without incremental commitment to each structure, competing material slows down the process, because it would lead to a combinatory explosion. Further results, however, relativize this account when it comes to the syntax-phonology interface (Ferreira and Swets, 2002). Incremental production is possible, but it is "under strategic control"; it depends on semantic information, and it could be modulated by external factors, such as stress.

If processing were fully incremental, then we would expect the most important, and perhaps the only, feature to affect when a memory retrieval occurred is when that word would be outputted. However, if other features modulate this, then it would imply that incremental processing is instead variable, as suggested by earlier accounts. 


\subsection{Speaking Rates}

A wide variety of empirical work has been done on speaking rates in general. We contrast our work from such studies due to our explicit model and connection with lexical memory, rather than finding empirical correlations. Still, studies showing the effects of frequency, recency, and context (Bell et al., 2009; Arnon and Priva, 2014) did motivate some of our modeling choices, as those are the key components of the ACT-R theory of memory. Moreover, the recent thrust of work on speech rates in general, such as those correlating it to information content (e.g. Arnon and Cohen Priva, 2013) also show an interesting empirical effect lacking a true cognitive explanation.

\subsection{Lexical Retrieval}

This paper examines the time course of lexical retrieval for the case of fluent, naturalistic speech. Lexical retrieval is known to experience interference, which provides evidence of the architecture: Schriefers et al. (1990) found that semantic, but not phonological material can cause interference, suggesting that the two are represented separately. This would imply that phonological information is handled somewhat separately than semantic information. Ratcliff and McKoon's (1989) study focuses on sentence retrieval and found that even semantic information was retrieved in stages. Now, we seek to model the retrieval process in the context of fluent speech. The prevailing model of lexical access divides the task into retrieving a lemma and retrieving the word's phonological form (Levelt, 1992). Little progress has been made toward explaining the process by which these retrievals interleave with the output of a sentence, given limited verbal working memory. The problem of the capacity, span and nature (modality) of verbal working memory is beyond the scope of this paper; however, it does set empirical constraints for how long before output each type of retrieval can happen (Baddeley, 2003). If there is interference far away from the output of the target word, then retrieving the phonological form of a word can happen well before it is outputted, or retrieving the lemma of the word could interfere with phonological encoding. It remains, even if there is interference, that some resources between phonology and the lexicon are shared, while others are separate.

A substantial body of work has focused on lexical access, which have likewise used and developed a variety of memory models to explain the data. In particular, we contrast our work with this due to our focus on fluent speech, rather than picture-naming. For instance, classic models such as Dell's (1986)'s model of spreading activation during language production and Levelt et al.'s (1999) WEAVER ++ model both rely on picture naming tasks, rather than fluent speech. While other models do use fluent speech, they focus on modeling errors rather than modeling speaking rates (e.g. Rapp and Goldrick, 2000).

More relevantly, Dell and O'Seaghdha (1992) also examine the time course of lexical access in language production. In particular, they use series of three words and EEG data to estimate lexical retrieval time. However, the lab setting it took place in precluded it as a study of naturalistic speech. Further, their 
model of the affects of word-properties relied on primarily qualitative attributes, such as semantic or phonetic relatedness. In particular, they find additional evidence for lemma and phonological retrieval happening in separate stages, based on inhibition and facilitation effects. The goal of the present study is to expand the examined time frame in the hopes of replicating their argument on naturalistic speech while viewing effects found throughout, rather than just a three word window.

\section{ACT-R Model}

To motivate the corpus-based empirical analysis, we first convey the highlevel model that describes the language production process. Our method primarily relies on simulating the state of lexical declarative memory during language production. After we simulate the memory retrievals for each word, we can compare this information to the actual empirical timing data in the corpus. In particular, we rely heavily on Anderson's (1983) original account of memory. This framework was selected rather than newer or more task-specific frameworks as it is the same underlying memory model of ACT-R, which has been used to explain a wide variety of language phenomena (e.g. Vasishth and Lewis, 2004; Reitter et al., 2009), but also has been used to explain everything from decision-making (e.g. Marewski and Mehlhorn, 2011) to visual attention in graphical user interfaces (e.g. Byrne et al., 1999). Thus, by using this model, our work naturally integrates with a large body of work, using the same mechanisms to explain a variety of tasks.

Figure 1 illustrates how lemma retrieval of a target word affects phonological encoding of speaking of an earlier word. Retrieval timing is computationally estimated using the cognitive architecture ACT-R, and we assume that this retrieval time proportionally affects phonological encoding. This can take place strategically, via a metacognitive process that coordinates these different modules, or via interference because both processes share declarative memory resources.

Our model of lexical memory is principally based on Anderson (1983)'s idea of recency, frequency, and context effects. Activation $(A)$ within the context of the ACT-R system is generally described by the sum of base-level learning (bll) and spreading activation ( $s a$ ), which we adopt for our model as well (Anderson et al., 2004). In general, one can think of our model of lexical memory as an emulation of an ACT-R model; however, our model traces the corpus but does not make decisions, so should not be thought of as a complete cognitive model. Activation, can be defined as a linear combination of spreading activation baselevel learning:

$$
A(x)=s a(x)+b l l(x)
$$

For our purposes, we consider $x$ to refer to an individual word. Base-level learning refers to the frequency and recency effects. In the base-level learning equation, it can refer to both because of the decay parameter, $d$, which causes 
more recent presentations to be more important, with older presentations (signified by their time of presentation, $t$ ) becoming exponentially less relevant. These older presentations, when considered together, add to the equation through their sheer quantity, providing the frequency effect, defined as:

$$
\operatorname{bll}(x)=\log \left(\sum_{i \in P_{x}} t_{i}^{-d}\right)
$$

In this equation, $P_{x}$ refers to the list of $x$ 's presentations, so $t_{i}$ is the time from that presentation to the present. Naturally, for something with as many presentations as any given word, it's infeasible to computationally manage that sum. However, the full equation can be approximated using only the total number of presentations and the $k$ most recent presentations and $n_{x}=\left|P_{x}\right|$ (Petrov, 2006).

$$
b l l(x) \approx \log \left[\sum_{i}^{k} t_{i}^{-d}+\frac{\left(n_{x}-k\right)\left(t_{n_{x}}^{1-d}-t_{k}^{1-d}\right)}{(1-d)\left(t_{n_{x}}-t_{k}\right)}\right]
$$

While Petrov (2006) shows that the equation is close even for $k=1$, we used $k=5$ to more closely approximate the original equation. We then use the ACT-R default for the decay parameter, 0.5. Note that while it's been suggested (e.g. Lewis and Vasishth, 2005) that this decay parameter could be different for language processing, in this work, we are only concerned with its relative, rather than absolute values.

In order to compute the total number of presentations, we relied on a fairly simple estimate. We multiply the number of seconds a person has been alive with the average speaking rate and that word's frequency to obtain an estimate of the amount of times a person has encountered that word. This is obviously a rough estimate, as it is difficult to measure the difference between being exposed to the lexical form of the word compared to the phonological form, and it is even harder to measure any subsymbolic exposure due to thought. Still, using this formula, a unigram score computed by SRILM (Stolcke, 2002) applied to the British National Corpus, the average speaking rate of Switchboard participants (197 words/minute) as computed by Yuan et al. (2006), and the average age of Switchboard participants (37) (Godfrey et al., 1992), we can detertmine a baseline number of presentations for every word in Switchboard.

Next, computing spreading activation on a corpus as described in Anderson (1983) would likewise be computationally intractable. However, Pirolli et al. (2005) showed that for large sample sizes of language, Pointwise-Mutual Information is nearly identical. Therefore, we use Semilar's PMI database computed on the Wikipedia corpus (Rus et al., 2013; Church and Hanks, 1990). In the ACT-R system, generally only items currently in working memory affect memory retrievals (Anderson et al., 2004). Likewise, we maintained the $n$ previous words in a buffer to compute their spreading activation to the next word. We used $n=5$ as a reasonable estimate for working memory size in language, as found in a reading task (Daneman and Carpenter, 1980), though we leave to 
future work whether this is the most accurate way to compute spreading activation in discourse. For our model, we compute the spreading activation between retrieved word, $x$, and each word in working memory, $y$, as:

$$
s a(x) \approx \sum_{y}^{n} p m i(x, y)=\sum_{y}^{n} \log \frac{p(x, y)}{p(x) p(y)}
$$

Once we have a value for activation, it's fairly simple to compute an estimate for retrieval time (RT) using the same equations from Anderson (1983).

$$
R T=I+1 / A-\frac{K e^{-K A}}{\left(1-e^{-K A}\right)}
$$

In this equation, $I$ is an intercept, easily fitted with a linear model. As a parameter, $K$ represents the cutoff time (in seconds) before there is a retrieval failure. Thus, this equation only represents successful retrievals, but due to our methodology, we can assume that every word was successfully retrieved, so it is appropriate. To our knowledge, no one has attempted to estimate this parameter for the task of language production, so we chose 1.0 as a default for $K$.

\section{Methods}

\subsection{Corpus Analysis}

The empirical speech data was taken from the Switchboard corpus (Godfrey et al., 1992) which is part of the Penn Treebank corpus (Marcus et al., 1993). This dataset consists of telephone conversations between strangers on a random topic, annotated to include the start and finish time for every word that has been spoken. Using our model of lexical memory as described in the previous section, we trace through the model and compute the activation of each word at its onset time.

Once the activation was computed for each word at the point when it was spoken, our goal was to observe its effect on overall speaking rates. In order to estimate when $x$ was retrieved, we examined the speech some number of words back from word $x$. If words are spoken systematically more slowly or quickly based on word $x$ 's activation and their positional relationship to word $x$, then we can assume where words are spoken more slowly, retrievals are taking place. Where words are spoken more quickly, retrievals have finished. Importantly, since this is being computed at every sentence position, this should not capture positional effects.

Our analysis of the corpus requires computing each word's delay, which is defined as the amount of time between the onsets of the two words, including any disfluencies that occur. As words themselves naturally can require different amounts of time to speak, we instead use the adjusted delay which is computed by taking the average of all of the durations of that word (as found in Switchboard) and subtracting it from the given duration. Thus, the adjusted delay 
could be a positive or a negative number. A positive number would represent a slowdown, and a negative number would represent a speedup.

These speedups and slowdowns, and their relationship to retrieval time, allow us to make an argument about the interaction between lexical and phonological processing. From a statistical point of view, as we are comparing retrieval time and slowdowns in the same units, our linear model could be thought of as the percentage of retrieval time that is behaviorally reflected in language production.

\subsection{Experiment}

Data were analyzed in two separate ways: an interaction model and a discrete model. For both models, the activation of a target word and its expected retrieval time burden was computed, as were the delays for the $n$ words preceding the target word. (Importantly, note that in both models, when we refer to the expected retrieval time or activation, we are referring to the target word, not any of the preceding words.) Both models are concerned with the index $i$, which refers to the number of interceding words between the given delay and the target word, such that $\mathrm{i}=0$ refers to the word immediately before the target word.

In the interaction model, we are interested in the interaction term between index and delay: its goal is to show how the correlation changes with index. In this model, every observation only uses a single index, chosen randomly, for each target word. All of the other indexes for that word are discarded. This is to ensure the observations are independent. The correlation coefficients of interest are the correlation of delay as a whole, and its interaction effect with index. In general, the coefficient of index by itself is likely capturing some distributional information about the data, rather than anything interesting with how it relates to memory retrievals. As a linear model, that would be:

$$
R T \sim \text { delay } * \text { index }
$$

Meanwhile, the discrete model's observations consist of a word and all of the previous indexes. Then, we make a linear model using each of the delays as a predictor, with each index again representing how many interceding words there are between it and the target word. As a linear model, that would be:

$$
R T \sim \text { delay }_{0}+\text { delay }_{1}+\ldots+\text { delay }_{n}
$$

The goal of the interaction model is to show the robustness of the slope associated with index, while the goal of the discrete model is to allow for a nonlinear relationship between index and the effect of delay on activation, examining up to 25 previous words.

Under the model shown in Figure 1, we expect that longer retrieval times of the target word are associated with slowdowns of speech production at some time before the target word is spoken. Earlier than that point, the target word should have no influence on speech production. 


\section{Results and Discussion}

Our experiments have yielded a relatively counterintuitive result: namely, delay is correlated in the direction opposite to what is expected. One would imagine that delay and retrieval time should be positively correlated: if people are speaking words more slowly (positive delay), then likewise, their retrieval time should be higher. However, we discovered a robust effect in the opposite direction: higher delays imply shorter expected retrieval times, and shorter delays imply longer expected retrieval times. In other words, when people are expected to need the longest to retrieve words, they speak more quickly, and vice versa. Naturally, this effect is hard to justify, but it can start to make sense once you remember that expected retrieval time, ultimately, is a product of activation, which could affect people's strategies for retrieval. Of more immediate importance is the interaction effect, which is in the expected direction.

Looking further back into the past, we see a pretty clear trend. The effect gradually changes direction, and we see shorter delays correlated with faster expected retrieval. This reliable interaction suggests that either there are two separate effects, or as we discuss later, one time-sensitive effect. We accept that in the discrete model, adjacent words' delays are collinear. Nonetheless, we still see a fairly clear trend as the effect passes through zero, passing from negative to positive, and then back to zero as the index becomes too far away to be relevant. See also Figure 2, which shows how the relationship between activation of a word and speech delay develops over the indexes, $i$, before the word, defined as:

$$
y(j, i)=\frac{A_{j}-\beta_{0}}{\operatorname{delay}_{i}}
$$

These graphs were designed to demonstrate how the effect switches from positive to negative as we move back from immediately before the word to earlier in the utterance. With the interaction model, we wanted to clearly show the statistical validity of the pattern of effects; with the discrete model, we wanted to make the gradual fade to zero obvious. Interpreting the two models should make the following three points clear:

1. There is a strong negative correlation of the word delays with expected retrieval time for the words immediately before the target word. Since retrieval time is a function of activation, this would imply that the observable phonological effect happens later for more activated words, which are likely retrieved shortly before their use.

2. There is a weaker but significant positive correlation of the word delays with expected retrieval time for words about 4-12 words preceding the target word. These delays likely occur for words with less activation, whose retrievals are likely initiated early to ensure that there is enough time.

3. For words very far away from the target word, there is no reliable effect, implying that this is not just an effect of a cyclical information distribution. 
Table 1: The linear effects model relating each discrete delay term with expected retrieval time. A higher number on the delay term signifies the number of words between the delayed word and the target word. See also Figure 3.

\begin{tabular}{|c|c|c|c|c|c|}
\hline & Estimate & Std. Error & t-value & \multicolumn{2}{|l|}{$\mathrm{p}$-value } \\
\hline $\mathrm{I}$ & .1844 & .0002 & 1203.52 & $<.00001$ & $* * *$ \\
\hline $\mathrm{d} 0$ & -.0033 & .0001 & -44.44 & $<.00001$ & $* * *$ \\
\hline $\mathrm{d} 1$ & -.0011 & .0001 & -14.28 & $<.00001$ & $* * *$ \\
\hline $\mathrm{d} 2$ & -.0006 & .0001 & -8.05 & $<.00001$ & $* * *$ \\
\hline $\mathrm{d} 3$ & -.0002 & .0001 & -1.96 & .0504 & \\
\hline $\mathrm{d} 4$ & -.0001 & .0001 & -1.78 & .0757 & \\
\hline $\mathrm{d} 5$ & -.0001 & .0001 & -0.64 & .5211 & \\
\hline $\mathrm{d} 6$ & .0002 & .0001 & 2.00 & $.0453 *$ & \\
\hline $\mathrm{d} 7$ & .0001 & .0001 & 1.30 & .1927 & \\
\hline $\mathrm{d} 8$ & .0002 & .0001 & 2.38 & $.0175 *$ & \\
\hline $\mathrm{d} 9$ & -.0001 & .0001 & -0.67 & .5053 & \\
\hline $\mathrm{d} 10$ & .0002 & .0001 & 2.98 & $.0029 * *$ & \\
\hline $\mathrm{d} 11$ & .0000 & .0001 & 0.27 & .7877 & \\
\hline $\mathrm{d} 12$ & .0002 & .0001 & 2.37 & $.0176 *$ & \\
\hline $\mathrm{d} 13$ & .0000 & .0001 & 0.56 & .5786 & \\
\hline $\mathrm{d} 14$ & .0001 & .0001 & 0.72 & .4714 & \\
\hline $\mathrm{d} 15$ & .0000 & .0001 & 0.38 & .7013 & \\
\hline $\mathrm{d} 16$ & .0000 & .0001 & 0.42 & .6721 & \\
\hline $\mathrm{d} 17$ & .0001 & .0001 & 1.66 & .0962 & \\
\hline $\mathrm{d} 18$ & .0000 & .0001 & 0.11 & .9143 & \\
\hline $\mathrm{d} 19$ & .0001 & .0001 & 1.15 & .2516 & \\
\hline $\mathrm{d} 20$ & -.0001 & .0001 & -0.77 & .4405 & \\
\hline $\mathrm{d} 21$ & .0000 & .0001 & 0.45 & .6545 & \\
\hline $\mathrm{d} 22$ & -.0001 & .0001 & -1.00 & .3188 & \\
\hline $\mathrm{d} 23$ & .0000 & .0001 & 0.06 & .9562 & \\
\hline $\mathrm{d} 24$ & .0000 & .0001 & 0.65 & .5174 & \\
\hline F-stat & DF & p-value & $\operatorname{Adj} R^{2}$ & Multi $R^{2}$ & \\
\hline 118.5 & 777903 & $<.00001$ & 0.0038 & .0038 & \\
\hline
\end{tabular}


Table 2: Linear regression predicting expected retrieval time of a target word as a function of the delay in speaking of a previous word at that index

\begin{tabular}{c|cccc} 
& Estimate & Std. Error & t-value & p-value \\
\hline & & & & \\
(Intercept) & .1796 & .0002 & 728.715 & $<.00001 * * *$ \\
index & .0015 & .0010 & 1.1512 & $.011^{* *}$ \\
delay & .0668 & .0048 & 13.839 & $<.00001^{* * *}$ \\
index:delay & -.0066 & .0005 & -12.411 & $<.00001 * * *$ \\
& & & & \\
\hline & & & & \\
F-stat & DF & p-value & Adj $R^{2}$ & Multi $R^{2}$ \\
102.2 & 802055 & $<.00001$ & 0.0003 & .0003
\end{tabular}

\section{Discussion}

The most important way to perceive expected retrieval time is as a function of activation. Activation makes less sense in the context of a linear model, since it refers to a log-probability, but recall that activation is the inverse function of retrieval time, and vice versa. In other words, higher activation implies a shorter expected retrieval time.

\subsection{Process Model}

We found a surprising effect: words with higher activation are not spoken more quickly, but more slowly. This also applies to the words that immediately precede them. However, if we look further back, we see a robust effect in the expected direction: if the approaching word has a high activation, they are said more quickly, but if the approaching word has a low activation, they are said more slowly. We argue that this slowdown is the result of shared resources between phonological and grammatical encoding, and as activation directly predicts retrieval time, we posit that word retrievals are part of what causes slowdowns. The corresponding speedups could be because the work of planning the sentence up to that point is then done. The most important prediction of this is that it means low activation words are retrieved earlier, which would imply that there is some cognitive strategy facilitating the necessity of initiating early retrievals for low activation words.

\subsection{Incrementality}

These results provide information about the timing of memory retrievals, given that such retrievals are related to activation. As activation is inherently related with how long a memory retrieval should take, it makes sense there are some cognitive strategies for coping with this disparity in order to produce 


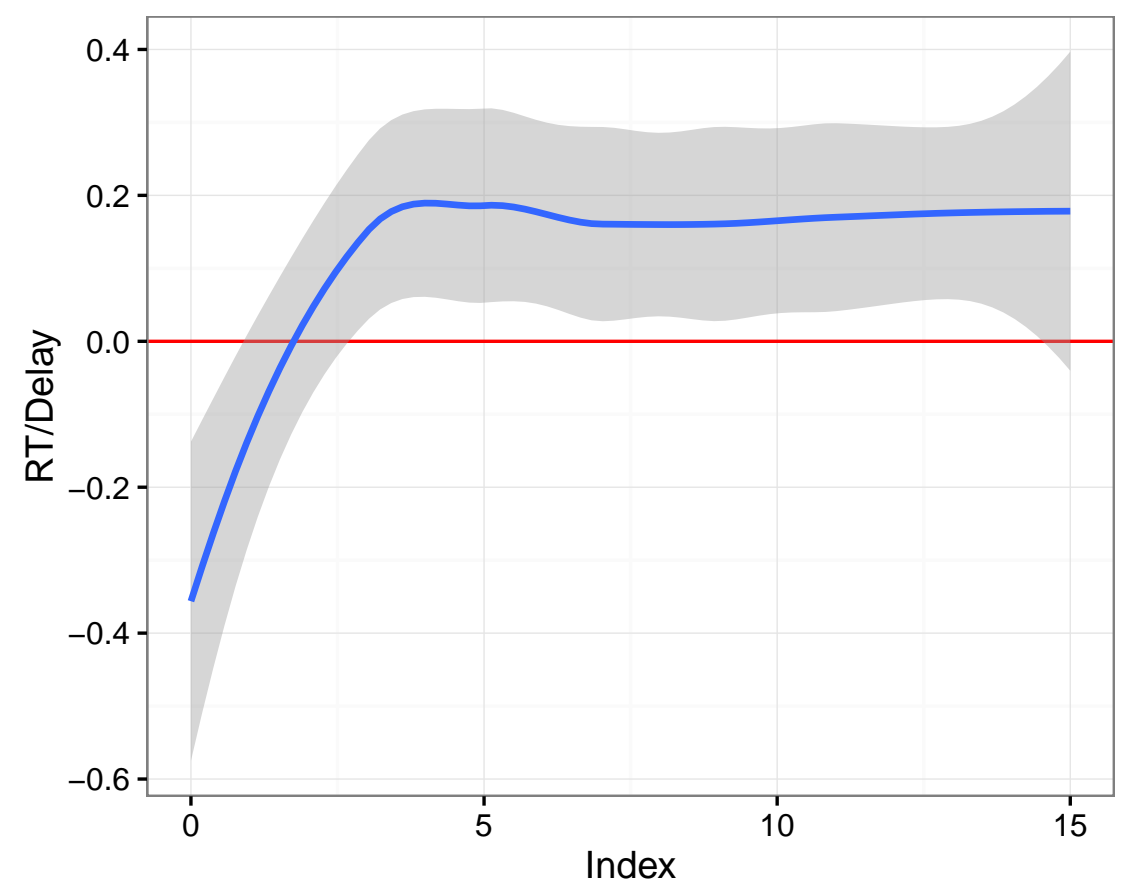

Figure 2: A graph of the correlation effects of delay and expected retrieval time across index, created using a sample of raw data. Index gives distance in words (up to 15) between observed delay and word determining activation.

seemingly fluent dialogue. That strategy involves buffering: retrieving and storing the words that will need longer to retrieve, based on the structure of the sentence.

Further, this type of buffering strategy could be part of the strategy that Ferreira and Swets (2002) refer to, when they propose the incrementality of language production is under "strategic control." While a purely incremental strategy might have interlocutors retrieve in a purely incremental fashion, there are some hiccups: certain words take longer to retrieve than others. By this logic, if grammatical encoding proceeds in a purely incremental fashion, than lexical retrieval does not, and vice versa. Thus, it is reasonable to believe that the grading of incrementality found in natural human discourse is not only variable from situation to situation, but it may be variable amongst competing processes for any given situation.

\subsection{Lexical Retrieval}

It is difficult to separate the lexical retrievals we found into the two retrievals described by Levelt (1992): a lemma retrieval and a later phonological retrieval. An implied assumption is that lemma retrievals shouldn't interfere with phonological processes (e.g. Schriefers et al., 1990), though it is difficult to know if 


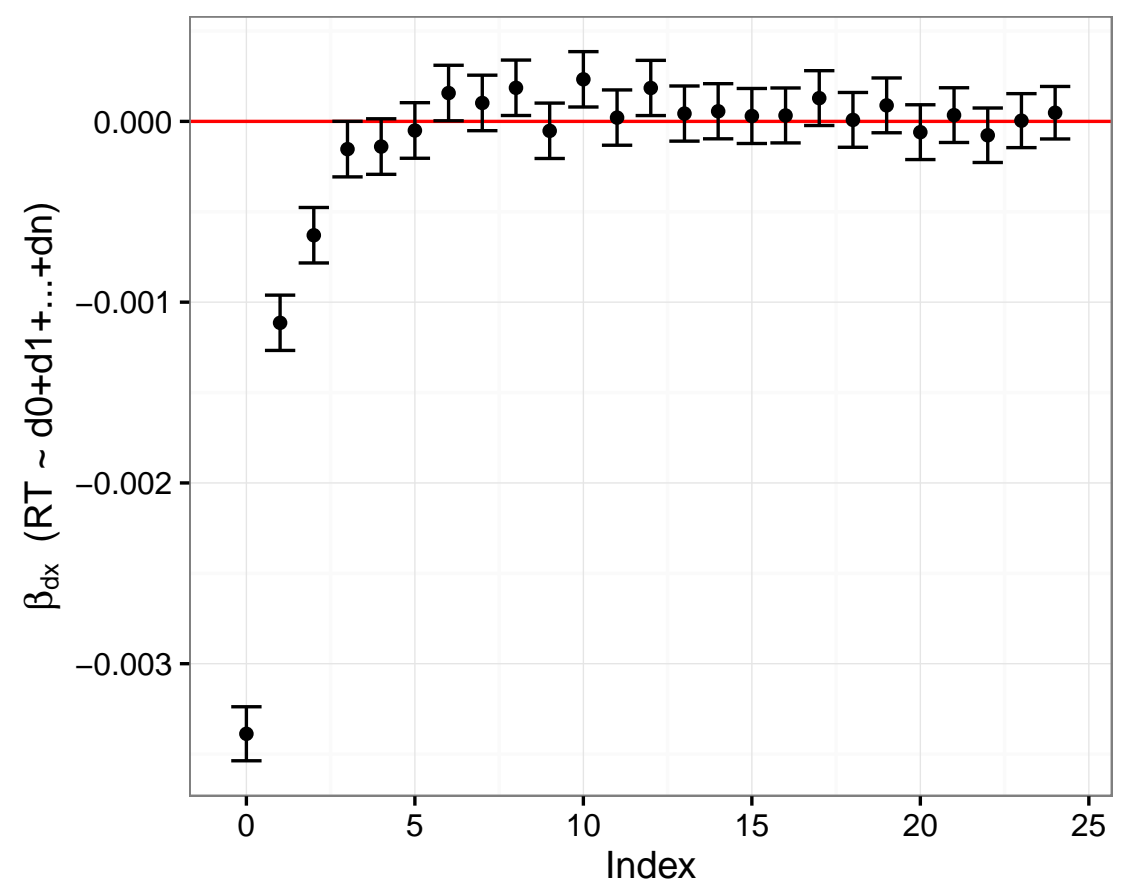

Figure 3: The discrete model's discrete predictors (see Table 1), by index. Error bars represent normal $95 \%$ confidence intervals.

a speech slowdown is due to a phonological or semantic interference. However, since effects are still observed at large distances from the target words, either phonological forms can be retrieved in a non-incremental way (possibly even before lemmas for other words are retrieved), or the retrieval of the lemma does interfere with phonological encoding in some way. Still, we ultimately find the same pattern of effects as Dell and O'Seaghdha (1992): facilitatory effects close to the target word, with inhibitory effects further away. The primary difference is the time frame, which is possibly due to their experimental setup.

\subsection{Uniform Information Density}

Let's consider an additional explanation. The Constant Entropy Rate Hypothesis (Genzel and Charniak, 2002) posits that lexical material is distributed across a sentence (and other units) such that its information is held approximately constant. Could a difficult-to-retrieve, slow word at position $j$ be likely to be combined with easier-to-retrieve, high-frequency words at positions $j-4 \ldots j-1$, causing the significantly increased speech rate we found there?

The model of buffered retrievals, along with the empirical evidence, may provide a cognitive mechanism that results in an approximately constant entropy rate. Thus, Uniform Information Density (UID, e.g., Jaeger, 2010) is seen less 
as a strategy to make optimize communication, than as a consequence of the cognitive procedures involved in retrieving syntactic-lexical items from declarative memory while grammatically encoding those materials retrieved earlier.

Importantly, we do not find a repetitive pattern of delays. The short positive correlation between activation of the target word and speed-up three words before the target word is not cyclical. This implies while memory retrievals could likely explain part of the UID hypothesis, the inherent distribution of information within a sentence does not by itself explain our results.

\section{Future Work}

Our work leaves open several possible avenues for future research. In particular, we are interested in how much of the uniform information density hypothesis can be explained by memory retrievals as a mechanism. While it is unclear if syntax rules are subjected to memory retrievals (see (e.g. Reitter et al., 2011) for a discussion), various features of fluent language production are. The use of syntax could, potentially, adapt to what is currently in working memory, rather than be the driving mechanism. By this argument, memory retrieval is a largely automatic, rather than attention-driven process, and syntax makes use of what it can to produce fluent dialogue.

Additionally, while we rely on the empirically validated ACT-R architecture to compute our activation, there is no guarantee that this is the best possible mechanism. Other metrics, such as surprisal (Hale, 2001), may in fact better predict memory retrievals. We consider the relative separation between information-theoretic linguistics and cognitive linguistics to be both important and addressable by future work.

Furthermore, this paper does little to address the actual distinction between lemma and phonological retrieval. Although we do not expect phonological forms to be retrieved as early as the effects we are seeing, we also do not expect lemma retrieval to have effects on phonological encoding. This contradiction we also leave to future work.

Lastly, the data we use is ultimately noisy, as it comes from multiple speakers. Normalizing carefully by speaker would increase the strength of our effect, though ultimately, this source of noise just shows the strength of the observed effects.

\section{Conclusion}

In this paper, we explore the process of lexical memory retrieval in the context of language production. In contrast to previous work, we look at a corpus of natural speech rather than relying on single word retrievals in an experimental setting. This allowed us to observe how certain processes involved in fluent language production overlap. In particular, the results support a model according to which lexical retrievals can happen quite early. By using the formalism defined by the empirically-validated ACT-R framework, we show when 
memory retrievals are taking place through the effect on speaking rates. In particular, we see a strong and robust negative effect of activation on delay immediately before phonological encoding, i.e., high activation words are spoken more slowly. Knowing the time course and which resources are implicated

and shared in language production has implications for the psycholinguistics of language production.

\section{References}

Anderson, J., 1983. A Spreading Activation Theory of Memory. Journal of Verbal Learning and Verbal Behavior 22, 261-295.

Anderson, J. R., 1991. Cognitive architectures in a rational analysis. In: VanLehn, K. (Ed.), Architectures for Intelligence. Lawrence Erlbaum Associates.

Anderson, J. R., Bothell, D., Byrne, M. D., Douglass, S., Lebiere, C., Quin, Y., 2004. An integrated theory of the mind. Psychological Review 111, 1036-1060.

Arnon, I., Cohen Priva, U., 2013. More than words: The effect of multi-word frequency and constituency on phonetic duration. Language and Speech 56 (3), 349-371.

Arnon, I., Priva, U. C., 2014. Time and again: The changing effect of word and multiword frequency on phonetic duration for highly frequent sequences. The Mental Lexicon 9 (3), 377-400.

Baddeley, A., 2003. Working memory: looking back and looking forward. Nature Reviews Neuroscience 4 (10), 829-839.

Bell, A., Brenier, J. M., Gregory, M., Girand, C., Jurafsky, D., 2009. Predictability effects on durations of content and function words in conversational english. Journal of Memory and Language 60 (1), 92-111.

Bock, J. K., Levelt, W. J. M., 2002. Language production: Grammatical encoding. Vol. 5. Routledge, pp. 405-452.

Byrne, M. D., Anderson, J. R., Douglass, S., Matessa, M., 1999. Eye tracking the visual search of click-down menus. In: Proceedings of the SIGCHI conference on Human Factors in Computing Systems. ACM, pp. 402-409.

Caramazza, A., 1997. How many levels of processing are there in lexical access? Cognitive neuropsychology 14 (1), 177-208.

Caramazza, A., 2006. Lexical access in bilingual speakers: What's the (hard) problem? Bilingualism: Language and Cognition 9, 153-166.

Church, K. W., Hanks, P., 1990. Word association norms, mutual information, and lexicography. Computational Linguistics 16 (1), 22-29. 
Collins, A. M., Loftus, E. F., 1975. A spreading-activation theory of semantic processing. Psychological review 82 (6), 407.

Daneman, M., Carpenter, P. A., 1980. Individual differences in working memory and reading. Journal of verbal learning and verbal behavior 19 (4), 450-466.

Dell, G. S., 1986. A spreading-activation theory of retrieval in sentence production. Psychological review 93 (3), 283.

Dell, G. S., O'Seaghdha, P. G., 1992. Stages of lexical access in language production. Cognition 42 (1), 287-314.

Dell, G. S., Schwartz, M. F., Martin, N., Saffran, E. M., Gagnon, D. A., 1997. Lexical access in aphasic and nonaphasic speakers. Psychological Review 104 (4), 801-838.

Ferreira, F., Swets, B., 2002. How incremental is language production? Evidence from the production of utterances requiring the computation of arithmetic sums. Journal of Memory and Language 46 (1), 57-84.

Ferreira, V. S., 1996. Is it better to give than to donate? Syntactic flexibility in language production. Journal of Memory and Language 35, 724-755.

Ferreira, V. S., Slevc, L. R., 2007. Grammatical encoding. Oxford University Press, p. 453.

Genzel, D., Charniak, E., 2002. Entropy rate constancy in text. In: Proceedings of the 40th Annual Meeting of the Association for Computational Linguistics. Association for Computational Linguistics, pp. 199-206.

Godfrey, J. J., Holliman, E. C., McDaniel, J., 1992. Switchboard: Telephone speech corpus for research and development. In: IEEE International Conference on Acoustics, Speech, and Signal Processing (ICASSP-92). Vol. 1. IEEE, pp. $517-520$.

Guhe, M., 2007. Incremental Conceptualization for Language Production. Lawrence Erlbaum Associates.

Hale, J., 2001. A probabilistic earley parser as a psycholinguistic model. In: Proceedings of the second meeting of the North American Chapter of the Association for Computational Linguistics on Language technologies. Association for Computational Linguistics, pp. 1-8.

Jaeger, T. F., 2010. Redundancy and reduction: Speakers manage syntactic information density. Cognitive Psychology 61 (1), 23-62.

Jespersen, O., 1992. The philosophy of grammar. University of Chicago Press.

Levelt, W. J., 1992. Accessing words in speech production: Stages, processes and representations. Cognition 42 (1), 1-22. 
Levelt, W. J., Roelofs, A., Meyer, A. S., 1999. A theory of lexical access in speech production. Behavioral and brain sciences 22 (1), 1-38.

Lewis, R. L., Vasishth, S., 2005. An activation-based model of sentence processing as skilled memory retrieval. Cognitive science 29 (3), 375-419.

Marcus, M. P., Santorini, B., Marcinkiewicz, M. A., 1993. Building a large annotated corpus of english: The penn treebank. COMPUTATIONAL LINGUISTICS 19 (2), 313-330.

Marewski, J. N., Mehlhorn, K., 2011. Using the act-r architecture to specify 39 quantitative process models of decision making. Judgment and Decision Making 6 (6), 439.

Petrov, A. A., 2006. Computationally efficient approximation of the base-level learning equation in ACT-R. In: Proceedings of the seventh international conference on cognitive modeling. pp. 391-392.

Pickering, M. J., Garrod, S., 2013. An integrated theory of language production and comprehension. Behavioral and Brain Sciences 36 (04), 329-347.

Pirolli, P., Fu, W.-t., Chi, E., Farahat, A., 2005. Information scent and web navigation: Theory, models and automated usability evaluation. In: Proc. HCI International.

Rapp, B., Goldrick, M., 2000. Discreteness and interactivity in spoken word production. Psychological review 107 (3), 460.

Ratcliff, R., McKoon, G., 1989. Similarity information versus relational information: Differences in the time course of retrieval. Cognitive Psychology 21 (2), $139-155$.

Reitter, D., Keller, F., Moore, J. D., 2009. A cognitive model of syntactic priming.

Reitter, D., Keller, F., Moore, J. D., 2011. A Computational Cognitive Model of Syntactic Priming. Cognitive Science 35 (4), 587-637.

Roelofs, A., Meyer, A. S., Levelt, W. J. M., 1998. A case for the lemma/lexeme distinction in models of speaking: Comment on caramazza and miozzo (1997). Cognition 69 (2), 219-230.

Rus, V., Lintean, M. C., Banjade, R., Niraula, N. B., Stefanescu, D., 2013. Semilar: The semantic similarity toolkit. In: ACL (Conference System Demonstrations). pp. 163-168.

Schriefers, H., Meyer, A. S., Levelt, W. J., 1990. Exploring the time course of lexical access in language production: Picture-word interference studies. Journal of memory and language 29 (1), 86-102. 
Stolcke, A., 2002. SRILM-an extensible language modeling toolkit. In: Proceedings of the International Conference on Spoken Language Processing. Vol. 2. Denver, pp. 901-904.

Vasishth, S., Lewis, R. L., 2004. Modeling sentence processing in ACT-R. In: Proceedings of the Workshop on Incremental Parsing: Bringing Engineering and Cognition Together. Association for Computational Linguistics, pp. 8287.

Yuan, J., Liberman, M., Cieri, C., 2006. Towards an integrated understanding of speaking rate in conversation. In: INTERSPEECH. 